\title{
El ecoturismo como estrategia de fortalecimiento en las acciones de conservación ambiental: Un análisis regional en Los Tuxtlas, Veracruz, México
}

\author{
Gilberto Manuel González Kuk* Christoph Neger**
}

Universidad Nacional Autónoma de México (México)

\begin{abstract}
Resumen: La región de Los Tuxtlas es privilegiada por la existencia de múltiples ecosistemas que brindan servicios a las poblaciones locales y albergan una gran biodiversidad de importancia nacional. Ante ello, en 1998 se decreta la Reserva de la Biosfera de Los Tuxtlas, cuyo objetivo es preservar y desarrollar junto con la población estrategias de conservación, entre ellas, el ecoturismo. La presente investigación analiza desde un enfoque regional las contribuciones e impactos de esta actividad en materia de conservación ambiental. Para esto se aplican métodos etnográficos y se elabora una representación cartográfica, para poder comprender las conjeturas territoriales de esta actividad. Se pudo evidenciar que las iniciativas de ecoturismo han logrado generar impactos de pequeña escala en el nivel local, aunque existe todavía un potencial importante para aumentar el impacto positivo, si se lograse juntar los esfuerzos dispersos para integrarlos bajo una estrategia general a nivel regional.
\end{abstract}

Palabras Clave: Ecoturismo; Turismo Alternativo; Reserva Natural; Conservación Ambiental; Los Tuxtlas.

Ecotourism as a strengthening strategy in environmental conservation actions: A regional analysis in Los Tuxtlas, Veracruz, Mexico

Abstract: The Los Tuxtlas region in the south of the state of Veracruz is privileged by the existence of multiple ecosystems that provide services to local populations and host a great biodiversity of national importance. Therefore, in 1998 the Los Tuxtlas Biosphere Reserve was decreed, whose objective is to preserve and develop conservation strategies together with the population, including ecotourism. This research analyses from a regional perspective the contributions and impacts of this activity in the field of environmental conservation. This is reached by the application of ethnographic methods and the elaboration of a cartographic representation, in order to understand the territorial conjectures of this activity. It could be shown that the ecotourism initiatives have managed to generate small-scale positive impacts on the local level. However, there is still potential to increase this impact, if the disperse efforts would be integrated into a general strategy on the regional level.

Keywords: Ecotourism; Alternative Tourism; Natural Reserve; Environmental Conservation; Los Tuxtlas.

\section{Introducción}

Los Tuxtlas, una región ubicada en el estado de Veracruz, en el sureste mexicano (Véase, Figura 1) es el reflejo de un territorio que ha sido asediado por diferentes problemáticas socioambientales a lo largo de la historia, condicionando la existencia de sus ecosistemas y erosionando los diferentes paisajes que ahora son relictos de lo que una vez fue una de las selvas tropicales más dominantes de México. La región se ubica al sureste del país, a $140 \mathrm{~km}$ del puerto de Veracruz.

Esta región representa una de las cinco regiones con mayor endemismo de árboles en México (con presencia de 26 de las 41 especies arbóreas exclusivas de las selvas húmedas de la vertiente del Golfo de México y del Caribe), considerándose un banco de germoplasma y conservación para especies de importancia ecológica (Paré \& Fuentes, 2007). En ella se han registrado 15 tipos de vegetación distintos,

Universidad Nacional Autónoma de México (México); E-mail: gmgonzalez@comunidad.unam.mx; https://orcid.org/00000003-4195-2086

** Universidad Nacional Autónoma de México (México); E-mail: neger@igg.unam.mx; https://orcid.org/0000-0001-5210-5005 
entre los cuales destacan: bosques mesófilos de montaña, selvas húmedas altas, medianas y bajas, bosques de encinos y de coníferas, dunas costeras, y manglares (Ramírez, 1999).

Este hecho, apremió que en 1998 que se decretara por mandato federal una de las áreas naturales protegidas más representativas de la región planicie costera y golfo de México, "Reserva de la Biosfera de los Tuxtlas" (RBT), con una extensión de 155,122 ha. En esta reserva se han descrito 102 especies de mamíferos, 49 especies de anfibios, 109 especies de reptiles, 561 especies de aves, 437 especies de peces (incluyendo el área de arrecifes que acompaña la costa de Los Tuxtlas), 359 especies de lepidópteros, 133 especies de Odonatos y 530 especies de Papilionoideos (CONANP, 2006).

Pese a ello, en 50 años, Los Tuxtlas ha perdido más del 45\% de selva tropical (entre otros ecosistemas), debido a las diferentes actividades económicas y antropogénicas como la ganadería, la agricultura, la expansión urbana, etc. Tan solo en los últimos 35 años se han producido cambios tan abruptos como la pérdida de más de 53,000 ha de selva alta, modificando los paisajes y los ecosistemas con la introducción de más de 40,000 ha de pastizales cultivados para la ganadería (González, 2018). Estas alteraciones y desequilibrios ecosistémicos no solo impactan a la biodiversidad de flora y fauna en la región, si no que presentan repercusiones evidentes y directas para la sociedad de la región. Ejemplo de ello es la escasez de agua, para consumo humano como para fines agropecuarios en la temporada seca, especialmente en años de sequía severa a causa de condiciones climáticas (Barragán, 2019; Secretaría de Gobernación, 2019). Como se reporta en medios de comunicación, esto ha llevado a los campesinos del sector agropecuario en la región a emigrar y buscar empleo fuera de sus lugares de origen (Salazar, 2020). También es evidente en muchos lugares en la región la erosión de la tierra que disminuye la productividad de los terrenos de uso agrícola y ganadero; el fuerte uso de agroquímicos por su parte contamina los suelos y también los cuerpos de agua, importantes tanto para la pesca como para el turismo (Gómez, 2017).

Ante este panorama, una de las actividades que se avizora como una estrategia global, regional y local capaz de subsanar las necesidades económicas de las comunidades a través de la protección de sus recursos naturales, bajo un componente de sustentabilidad, que no comprometan la existencia de los recursos naturales para las próximas generaciones, es el "ecoturismo". Ceballos (1998) plantea que a través de esta actividad las comunidades pueden tener una alternativa para seguir viviendo de los recursos naturales bajo un uso más sustentable y de esa manera mejorar sus estándares de vida.

\section{Figura 1: Localización de la región de los Tuxtlas.}

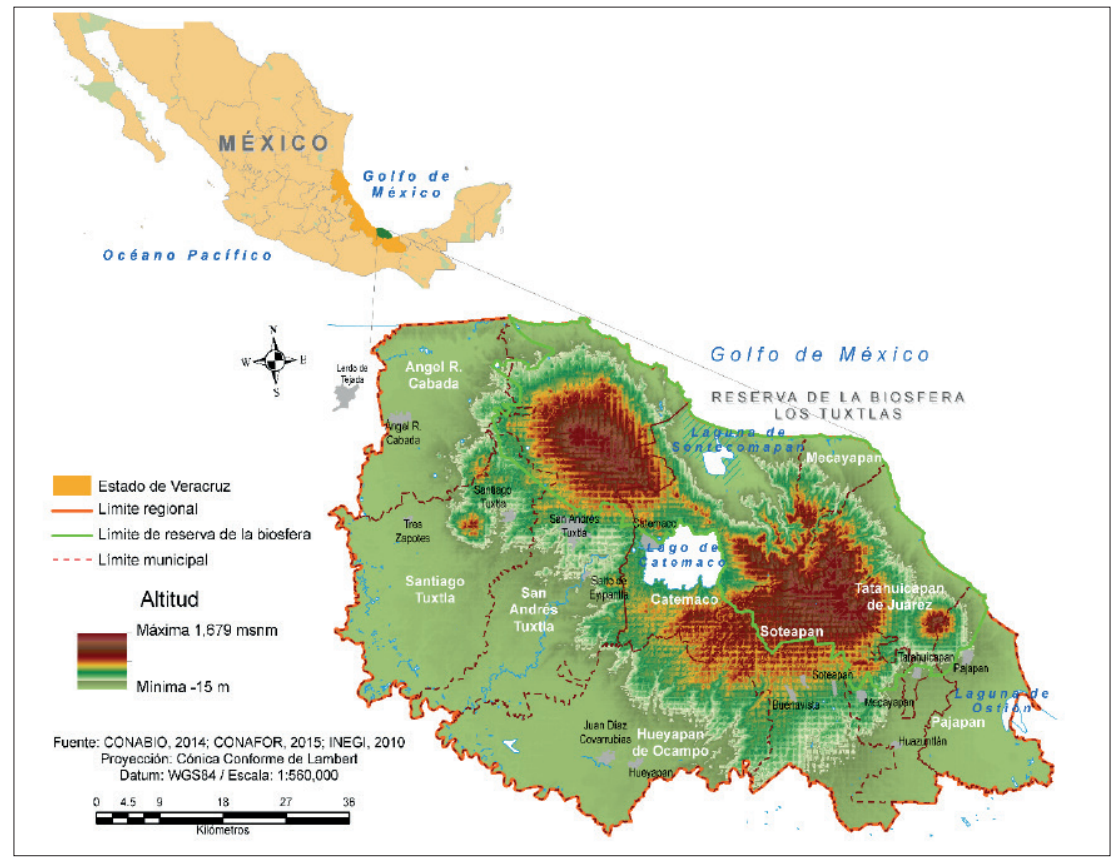

Fuente: Elaboración propia 


\section{Ecoturismo: conceptualización y praxis}

Mientras que los viajes a lugares naturales y, desde la creación del Parque Nacional Yellowstone en los EEUU en el 1872, a áreas naturales protegidas tienen una larga tradición (Orgaz, 2014; Rivera, 2010; Serulle, 1999), fue hasta los años 80 del siglo XX que se desarrollaba el concepto del ecoturismo. Si bien la palabra ecoturismo ya se había mencionado en una publicación en el 1965 (Higham, 2007), fue sobre todo en los años 80 que el prefijo "eco" cobraba importancia, indicando la relación de algo con el cuidado del medio ambiente (Puertas, 2006).

En este sentido, el ecoturismo puede ser entendido como un turismo de naturaleza que, además de apreciar los recursos naturales, contribuye a su conservación. Por ende, en el ecoturismo se fundamenta el componente ambiental a través de diferentes estrategias y acciones de conservación ambiental, ya que su finalidad no solo es la recreación, sino la preservación del medio natural (Fennell, 2008; Wearing, 1999) ${ }^{1}$. A esto se le agregan además otros aspectos, como se definió, por ejemplo, en la Cumbre Mundial del Ecoturismo que fue organizado por la ONU en el año 2002 en Quebec. El ecoturismo, de acuerdo con esta definición debe ser un turismo sustentable, con respecto a la dimensión económica, social y ecológica, que mejora el bienestar de las comunidades locales y las involucra en la planificación y el desarrollo de la actividad. Además debe interpretar el patrimonio natural y cultural del lugar visitado y ser de baja escala (OMT y PNUMA, 2002). Más adelante, se fundó la Sociedad Internacional de Ecoturismo (TIES por sus siglas en inglés), que resumió el concepto del ecoturismo como un turismo responsable en áreas naturales que conserva el ambiente y mejora el bienestar de las personas locales (Honey, 2008). De acuerdo a Fletcher (2018), en la actualidad ésta última sigue siendo la definición más ampliamente aceptada 2 .

Pese a estas definiciones, muchas veces existe confusión acerca del significado del término ecoturismo y, sobre todo, de su aplicación en la praxis (Orgaz y Castellanos, 2013). Por ello, muchos autores han criticado al ecoturismo de causar efectos nocivos al igual como el turismo convencional, o incluso peor por situarse en lugares con un medioambiente de alto valor y en muchas ocasiones sensibles a perturbaciones. Además se han reportado efectos negativos para las comunidades locales (Geffroy et al., 2017; Fletcher y Neves, 2012; Liu, 2003; Shannon et al., 2017; Voumard, 2019; Wearing y Neil, 1999). No obstante, como argumenta Honey (2008), los efectos negativos relacionados al ecoturismo en la mayoría de los casos son resultado de una aplicación errónea del término, sea por malentenderlo o por usar la palabra de ecoturismo de manera engañosa para promocionar ofertas turísticas que en realidad no tienen nada que ver con la definición científica del concepto.

Sin embargo, siguiendo a Honey, sería equivocado desechar el concepto del ecoturismo, solo porque ha sido aplicado en ciertos lugares de manera errónea, cuando al mismo tiempo hay muchos ejemplos de iniciativas de ecoturismo que sí cumplen con la definición de este concepto y han conllevado importantes efectos ambientales y sociales. A nivel mundial en este contexto se pueden mencionar el noreste de China (Fangfang et al., 2019), Botswana (Mbaiwa, 2015) y Ladakh, India (Vanelli et al., 2019) y la contribución directa a la conservación como en el caso de los cetáceos en Hawaii (Currie et al., 2018), de las áreas montañosas de Nepal (K.C., 2016) y de los felinos grandes en Africa (Mossaz et al., 2015).

En Latinoamérica Costa Rica en general se considera como el país ejemplar en cuanto al desarrollo ecoturístico en sus áreas naturales protegidas (Honey, 2008), aunque iniciativas exitosas también se han documentado, por ejemplo, con respecto a la conservación de guacamayas en Brazil (daSilva et al., 2018) y los bosques andinos en una comunidad del Ecuador (Huaraca et al. 2017); La Red Nicaragüense de Turismo Rural Comunitario (RENITURAL), conformada por 38 casos con manejo sustentable del territorio (Cañada et al., 2006); y diferentes casos en las regiones de Colombia donde se impulsa la observación de aves como recurso potencial para la educación ambiental y alternativa económica de la población local (Lara et al. 2016). También en México se han publicado relatos de experiencias exitosas del desarrollo del ecoturismo auténtico; se pueden mencionar ejemplos de la Selva Lacandona en Chiapas (de la Maza et al., 2015) y de la Sierra Norte de Oaxaca (Rosas-Baños y Correa-Holguín, 2016). Cada uno de estos modelos, integra bajo su propio esquema el desarrollo del "ecoturismo", considerando elementos como: su autodeterminación y reconocimiento étnico; la apropiación de sus territorios y la re-valorización y manejo de la naturaleza.

Dada las críticas al ecoturismo, que cuestionan si en sí es una actividad deseable en áreas naturales protegidas, también en el contexto de la huella de carbono generada por los viajes de los turistas (Buckley, 2009; Marzouki et al., 2012)-, o al menos si es conveniente utilizar recursos para la conservación para apoyar al ecoturismo (cp. Kiss, 2004), es importante seguir indagando en los efectos positivos en diferentes espacios. En este sentido, la presente investigación estudia los efectos de la actividad ecoturística para la conservación en un área donde ésta se ha insertado muy temprano, desde finales de los años 80, y donde hoy en día se encuentra un número importante de iniciativas de ecoturismo de diferente tamaño y forma de organización. 


\section{Marco metodológico para el análisis regional}

La aproximación metodológica conjugó una primera construcción de estado del arte sobre la región, de enfoques antropológicos y análisis geográficos (Bernard, 2011), y realizando trabajo de campo, bajo métodos etnográficos por un período de 10 meses entre 2017, 2018 y principios de 2019, con la aplicación de entrevistas semiestructuradas e informales, la participación de distintos actores locales (gubernamentales, sociedad civil, empresarios, etc.) y principalmente a las iniciativas de ecoturismo existentes de la región.

Por otra parte, se integró un método cartográfico, con la utilización de diferentes técnicas de análisis y representación espacial, a través del uso de los sistemas de información geográfica, para la caracterización del territorio y la representación de los resultados obtenidos del trabajo de campo. La aplicación de estos sistemas en el manejo de información y presentación de resultados data de un estricto proceso sobre el cual se definen desde la utilización de insumos vectoriales a utilizar, hasta los diferentes métodos de representación con respecto a la temática de la información (Olaya, 2014).

En esta investigación, se encontraron 30 iniciativas de ecoturismo de las cuales 5 dejaron de operar en los últimos años por conflictos de organización interna y en 2 su situación es indefinida, quedando al final 23 iniciativas al interior de la región de Los Tuxtlas, las cuales fueron punto de partida para el análisis regional (una iniciativa más se abandonó durante el tiempo del trabajo de campo, por lo que aun forma parte de esta investigación). Cabe mencionar que estas iniciativas surgen en diferentes años y se distribuyen entre 7 municipios, localizados en distintos paisajes, algunos de muy cercanos a las zonas núcleo de la reserva como se aprecia en la Figura 2.

Figura 2: Localización de las iniciativas de ecoturismo en la región de los Tuxtlas.

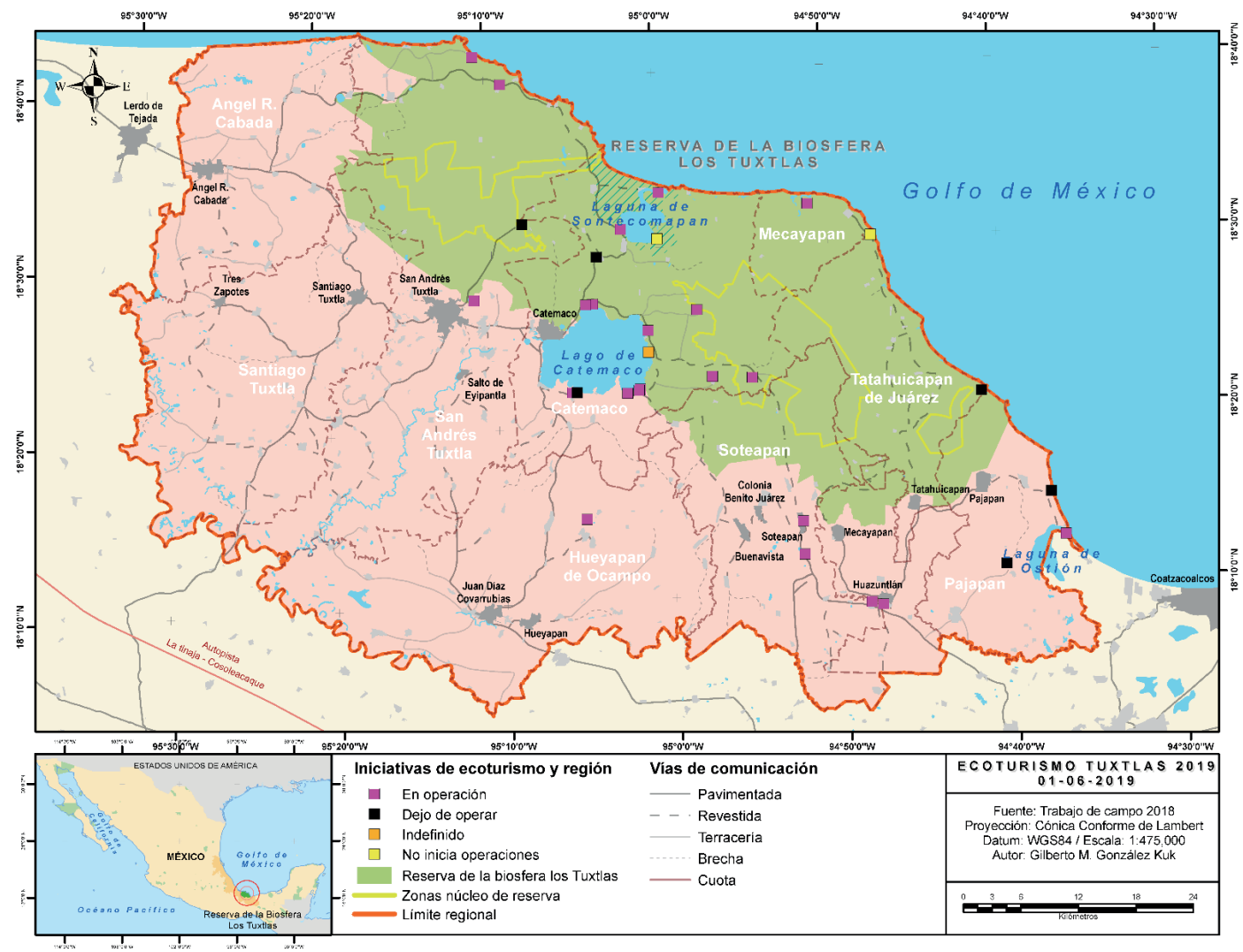

Fuente: Elaboración propia con base en el trabajo de campo. 


\section{Génesis del ecoturismo en Los Tuxtlas}

Los Tuxtlas se ha matizado bajo una identidad de "shamanería" o brujería, por más de 40 años, la cual se fundamenta desde el periodo prehispánico, concibiéndose como una "región etnoespacial" que Vargas (2004) describe como resultado de la convergencia en diferentes épocas entre zoques-popolucas, mixes y nahuas, que a través de sus cosmovisiones dieron una reconfiguración y sincretismo simbólico al territorio, el cual, con la llegada de los españoles en 1520 (incorporando la región a los dominios del marquesado con la introducción de cultivos de caña), traen consigo esclavos provenientes de África (Melgarejo, 1949), cuya cosmovisión se enraíza en la shamanería y los dioses místicos relacionados al mundo terrenal.

Este sincretismo y misticismo entre cosmogonías produjo una atracción relevante para historiadores y arqueólogos, misma que con el paso del tiempo posicionó a la región y específicamente al municipio de Catemaco, como "lugar de brujos"; incluso hoy, el folclore y las limpias asociadas a la práctica de la "shamanería" siguen vigentes (Vargas, 2004).

Pese a estos orígenes, el reconocimiento de Catemaco se impulsó bajo perspectivas orientadas al desarrollo turístico en la región, que años más tarde se posiciona aún más, gracias a la diversidad biológica existente, redirigiendo hacia los intereses conservacionistas en la región y buscando alternativas más sustentables, al modelo de turismo místico y convencional desarrollado. En este sentido, el ecoturismo en los Tuxtlas se abre paso a través de un turismo enfocado al misticismo, pero con trasfondo dirigido al modelo convencional. En los siguientes apartados se describe la inserción del ecoturismo en la región, con base en los trabajos de Díaz-Carrión y Neger (2014), González (2018), Juárez (2016), Lein (2011), Magio et al. (2017), Neger (2013; 2019) y Piñar (2011).

En la década de los 80's se inserta ecoturismo por medio de la reserva ecológica "Nanciyaga", proyecto privado que inició en el año de 1986, bajo un esquema familiar, el cual buscaba dar a conocer la selva y sus distintos ecosistemas a los visitantes del lago de Catemaco, protegiendo un remanente de selva tropical de 14 ha (propiedad privada) colindante a las orillas del lago y diversificando la oferta turística de la región, la cual se dirigía únicamente al sector de hotelería, restaurantes y paseo por el lago. Ante ello, esta iniciativa, a través de su objetivo de cuidar la selva, logró instaurar una nueva forma de recreación bajo un esquema de conservación, tal y como lo plantea el ecoturismo.

Posteriormente, en 1991, se lleva a cabo el denominado "Proyecto Sierra Santa Marta" (PSSM), el cual constó de la implementación de un diagnóstico de la región con el objetivo de determinar estrategias de desarrollo sustentable, puesto que, 7 años más tarde (en 1998) se decreta de manera oficial la RBT, lo cual limitaría el aprovechamiento de los recursos naturales a las poblaciones locales y establecería nuevas pautas de aprovechamiento y uso de los recursos naturales.

Figura 3: Inicio de operaciones de las iniciativas de ecoturismo en la región entre el periodo de 1980 a 2017.

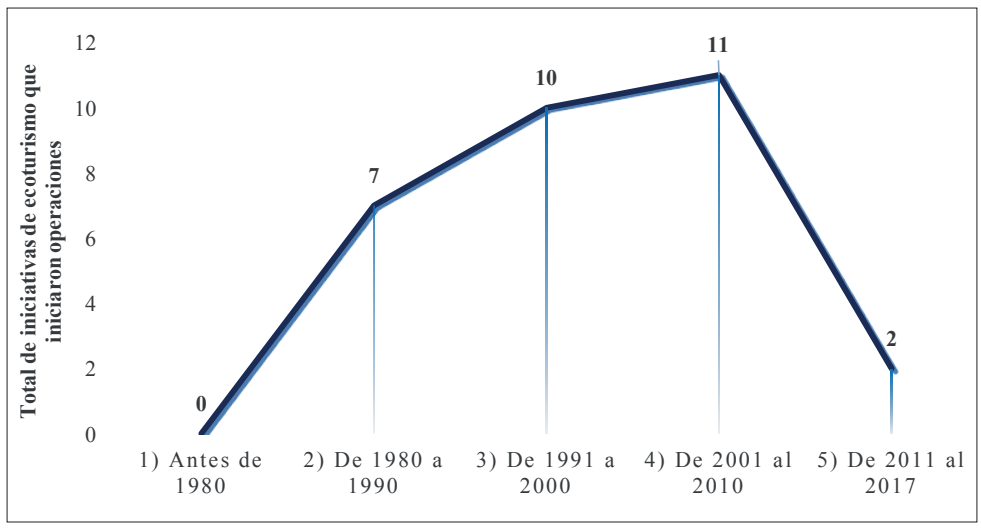

Fuente: González 2018. 
A través del PSSM, se desarrollan las primeras experiencias de ecoturismo comunitario durante el año de 1997, cuando se realiza la apertura de un modelo de "ecoturismo comunitario campesino" en el ejido de Adolfo López Mateos, una comunidad de alrededor de 100 habitantes, pionera en el desarrollo del ecoturismo en el contexto comunitario. Posteriormente a este pilotaje, se promovió el ecoturismo en otras comunidades, como son: Miguel Hidalgo con el proyecto "Apompal" y en Sontecomapan con el proyecto "Manglares de Sontecomapan". A consecuencia de estas primeras iniciativas, en el período del 2001 hasta al 2010, el ecoturismo en los Tuxtlas se posiciona como una nueva estrategia sobre el uso de los recursos naturales, siendo impulsado por actores locales (Véase, Figura 3), a través de distintas conformaciones jurídicas como cooperativas, grupos de trabajo ejidales, sociedad de solidaridad social, así como personas físicas que desarrollan proyectos de índole privada.

\section{Mecanismos para la conservación ambiental y su fortalecimiento a través del ecoturismo en Los Tuxtlas}

En Los Tuxtlas, se han implementado acciones locales sobre las cuales el ecoturismo encontró su florecimiento y ha sido sustento para su promoción; ejemplo de ello son las reservas ejidales donde seis de las iniciativas, a través de acuerdos ejidales, decretaron una fracción de sus ejidos a la conservación de la selva, con el objetivo de resguardar sus fuentes de abastecimiento de agua (manantiales, ríos, microcuencas, etc.) para sus actividades productivas. Cabe mencionar, que estas reservas ejidales fueron constituidas entre los años 1968 a 1980, mucho antes del decreto federal de la reserva de la biosfera, formando parte de las primeras acciones de conservación ${ }^{3}$ a través de las cuales se ha logrado rescatar y resguardar parte importante de la biodiversidad de la región.

Por otra parte, también existen las Unidades de Manejo Ambiental (UMA), políticas de conservación ambiental a nivel local establecidas por el Secretaria de Medio Ambiente y Recursos Naturales (SEMARNAT), las cuales han tenido una convergencia favorable con las actividades ecoturísticas y la protección de los recursos naturales ${ }^{4}$. Ante ello, algunas iniciativas han impulsado estas estrategias con la finalidad de proteger sus espacios donde operan su actividad ecoturística.

Las UMA en la región son impulsadas principalmente por las iniciativas privadas, ya que ven esta estrategia como una fuente clave para acceder a financiamientos y diversos apoyos para el impulso del ecoturismo. Sin embargo, el territorio protegido a través de la constitución de UMA refleja una franja muy escasa respecto a las reservas ejidales, donde estás últimas cubren cerca del $66 \%$ de la superficie bajo la que operan las iniciativas de ecoturismo. Sin embargo, tanto las UMA como las reservas ejidales, no son estrategias aisladas, ambas dan sustento a la conservación ambiental y en algunos casos las iniciativas congenian con ambas estrategias en sus sitios de operación (Véase, Figura 4).

De igual manera, estos mecanismos de conservación (reservas ejidales y UMA) no son las únicas acciones implementadas por las 26 iniciativas de ecoturismo en la región, cada una ha tomado distintas estrategias para conservar su área de operación, así como preservar la flora y fauna existente en la región. Entre las acciones elementales desarrolladas por las iniciativas están el manejo de sus residuos sólidos, con la separación de sus desechos; el uso de ecotecnologías (el 50\% de las iniciativas cuenta con ello); la impartición de educación ambiental a los visitantes ${ }^{5}$ (81\% de las iniciativas ofrecen talleres y explicación durante el recorrido, así como en ocasiones realizan talleres en escuelas y comunidades); y también realizan acciones de cuidado y protección de la flora y fauna de la región (Véase, Tabla 1), esto último es un factor relevante, ya que los integrantes de las iniciativas de ecoturismo, convertidos en custodios del medio ambiente, son ahora aliados clave para las estrategias alineadas a la conservación ambiental que se aplican en la reserva de la biosfera.

Cabe mencionar que, a pesar de las inconsistencias institucionales en materia ambiental y las políticas públicas desarrolladas en escalas macrorregionales o nacionales, estas iniciativas desde las esferas locales tratan de impactar positivamente con su manejo ambiental y resguardo de los recursos naturales. Sin embargo, también hay casos que han dejado de operar, especialmente en el sur de Los Tuxtlas, como en las comunidades Playa Linda, El Pescador y Peña Hermosa (observación de campo). El abandono de estas iniciativas, además de conflictos de organización interna, en muchas ocasiones se debe a que han permanecido vigentes a través de subsidios y al momento de no contar con ellos, se desarticulan, situación que ha sido una constante a nivel nacional ${ }^{6}$. 


\section{Figura 4: Mecanismos para la conservación ambiental impulsadas por las iniciativas de ecoturismo.}

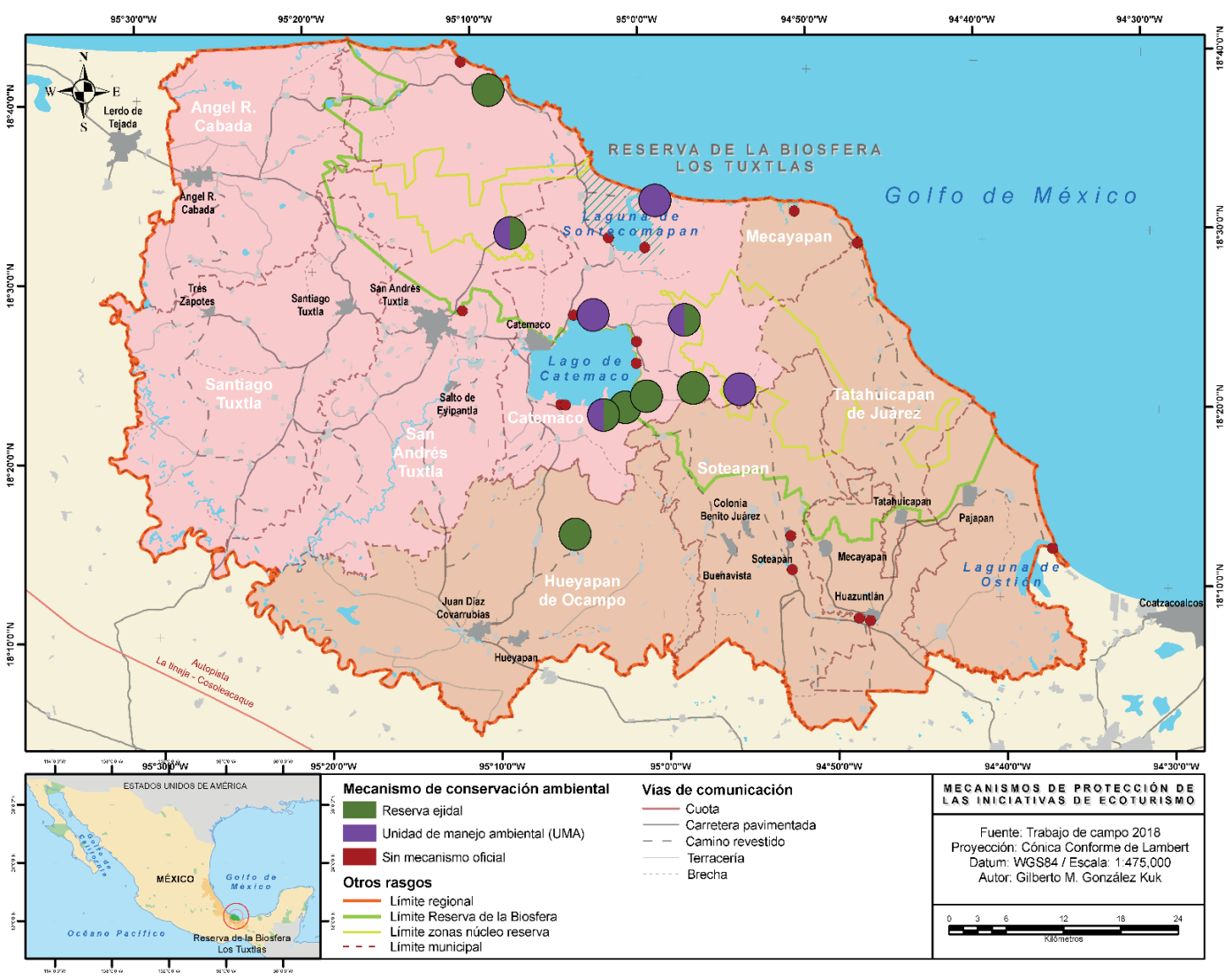

Fuente: González 2018.

En la Figura 4 se aprecia la localización de las iniciativas de ecoturismo y sus mecanismos de conservación ambiental, sustentado en políticas de resguardo y protección del territorio. Cabe mencionar que, aunque algunas cuentan tanto con UMA y reserva ejidal, un 57\% de las iniciativas no cuenta con algún mecanismo oficial para la protección de su territorio. En los casos de los ejidos estos acuerdos de conservación ambiental son tomados mediante las asambleas, formalizando un acuerdo de palabra entre los ejidatarios. Esto representa un punto de oportunidad para la formalización de los mecanismos de conservación y puedan ser considerados en las políticas de conservación nacional.

De igual manera, existen dos iniciativas de ecoturismo, las cuales aún no inician operaciones, sin embargo, ya realizan trabajos de adecuación en sus áreas de operación, así como han considerado capacitarse en temas referentes al cuidado y manejo de sus recursos naturales. Estas iniciativas son "Selvas y manglares del Toztlán" y "Centro ecoturístico Aati", estás iniciativas en 2018 aún se encuentran con preparativos para ofrecer sus servicios, entre los que se encuentran senderismo, recorridos en lancha, alimentación y acampado.

Por otra parte, las iniciativas presentadas realizan sus acciones de conservación de forma aislada, es decir, han intentado asociarse para crear una red o un mecanismo que pueda agruparlas y así promocionar tanto a la actividad ecoturística, como dar a conocer su aportación en materia ambiental, sin embargo, no ha sido posible, ya que la complejidad entre formas de operatividad, intereses y su condición entre actores privados y las comunidades rurales les ha impedido una correcta vinculación. 
Tabla 1: Acciones de conservación emprendidas por parte de las empresas de ecoturismo.

\begin{tabular}{|c|c|c|}
\hline No & $\begin{array}{c}\text { Iniciativa de } \\
\text { ecoturismo }\end{array}$ & Actividades de conservación \\
\hline 1 & Nanciyaga & $\begin{array}{l}\text { Conservación de } 14 \text { ha de selva, reintroducción de guacamaya roja Ara } \\
\text { macao ssp. cyanoptera y mono aullador Alouatta palliata ssp. mexicana; } \\
\text { UMA de cocodrilo de pantano Crocodylus moreletii e iguana verde Iguana } \\
\text { iguana. }\end{array}$ \\
\hline 2 & Selva del Marinero & $\begin{array}{l}\text { Conservación de reserva ejidal de } 125 \text { ha y otras áreas en el ejido con selva } \\
\text { (en total alrededor de } 300 \text { ha); arboretum de } 2 \text { ha; UMA de jabalí de labios } \\
\text { blancos Tayassu pecari. }\end{array}$ \\
\hline 3 & El Apompal & $\begin{array}{l}\text { Conservación de reserva ejidal de } 24 \text { ha; los socios también poseen áreas de } \\
\text { acahual; monitoreo de aves; reforestación de manantiales. }\end{array}$ \\
\hline 4 & $\begin{array}{l}\text { Manglares de } \\
\text { Sontecomapan }\end{array}$ & $\begin{array}{l}\text { Área de reforestación de } 1.5 \text { ha; participación en reforestaciones en } \\
\text { otras áreas; monitoreo de aves. }\end{array}$ \\
\hline 5 & $\begin{array}{l}\text { Ecoturismo } \\
\text { Arqueológico Las } \\
\text { Margaritas }\end{array}$ & Los socios poseen áreas de selva y acahual. \\
\hline 6 & Cascadas Encantadas & $\begin{array}{l}\text { Conservación de la reserva ejidal de } 35 \text { ha (compartida con la empresa } \\
\text { Anolis); los socios también poseen áreas de selva. }\end{array}$ \\
\hline 7 & Poza Reyna & Conservación de 20 ha de selva. \\
\hline 8 & Yambigapan & Área de reforestación de 1.5 ha. \\
\hline 9 & $\begin{array}{l}\text { Los Clarines } \\
\text { (Recientemente dejó } \\
\text { de operar) }\end{array}$ & $\begin{array}{l}\text { UMA de tepezcuintle Cuniculus paca; los socios poseen áreas de selva y } \\
\text { de reforestación, monitoreo de aves. }\end{array}$ \\
\hline 10 & Los Amigos & $\begin{array}{l}\text { Área de conservación y reforestación de } 40 \text { ha ( } 20 \text { ha de selva y } 20 \\
\text { ha de reforestación). }\end{array}$ \\
\hline 11 & Xococapan & Área de conservación y reforestación de 30 ha, monitoreo de aves. \\
\hline 12 & Jomxuk & Área de conservación y reforestación de 8 ha. \\
\hline 13 & Anolis & $\begin{array}{l}\text { Conservación de reserva ejidal de } 35 \text { ha; los socios también poseen áreas } \\
\text { de selva; reintroducción de la guacamaya roja Ara macao ssp. cyanoptera, } \\
\text { reforestación de } 400 \text { árboles; monitoreo de aves. }\end{array}$ \\
\hline 14 & Laguna del Ostión & Reforestación anual de selva y manglares (2017: 200 árboles). \\
\hline 15 & La Otra Opción & $\begin{array}{l}\text { Área de conservación y reforestación de } 115 \text { ha (50 ha de selva y } 65 \text { ha de } \\
\text { reforestación); UMA de palma de cola de pescado Chamaedorea ernesti- } \\
\text {-augustii de } 2 \text { ha, } 1 \text { plantación agroforestal de pimienta Pimenta doica } \\
\text { de } 15 \text { ha, UMA de hocofaisán Crax rubra, serete Dasyprocta mexicana, } \\
\text { tepezcuintle Cuniculus paca, jabalí de labios blancos Tayassu pecari y } \\
\text { temazate Mazama temama; monitoreo de aves. }\end{array}$ \\
\hline 16 & Ceytaks & Conservación de reserva ejidal de 57 ha. \\
\hline 17 & Kan Tasejkan & Área de reforestación de 3 ha. \\
\hline 18 & Los Arrecifes & $\begin{array}{l}\text { Reforestación de } 0.5 \text { ha de selva y } 3 \text { ha de dunas costeras; participación de } \\
\text { los socios en un campamento tortuguero. }\end{array}$ \\
\hline 19 & $\begin{array}{l}\text { Centro Etnoturístico } \\
\text { Tsabats Nas }\end{array}$ & Área de reforestación de 10 ha. \\
\hline 20 & $\begin{array}{l}\text { Centro Ecoturístico } \\
\text { Jem Takxi }\end{array}$ & Área de conservación de 10 ha. \\
\hline 21 & Eco Callí & $\begin{array}{l}\text { La superficie con la que cuentan es muy pequeña, de apenas } 1 / 4 \mathrm{ha} \text {, sin } \\
\text { embargo, es una de las iniciativas que más se ha capacitado en el manejo } \\
\text { y operación de las ecotecnologías, manejo de residuos sólidos, así como } \\
\text { impartición de talleres de educación ambiental en las comunidades }\end{array}$ \\
\hline 22 & El Teterete & Área de conservación de 1.5 ha; monitoreo de aves. \\
\hline 23 & Cascada El Tucán & Área de conservación de 3 ha. \\
\hline
\end{tabular}

Fuente: Elaboración propia con base en el trabajo de campo. 


\section{Contribuciones del ecoturismo a la conservación ambiental}

La contribución activa a la conservación del medio ambiente es la cualidad esencial para distinguir el ecoturismo dentro del concepto más general del turismo de naturaleza ${ }^{7}$. En este sentido, muchas de las empresas ecoturísticas mencionadas han hecho esfuerzos notables, como se aprecia en la Figura 4. Por otra parte, el impacto negativo del ecoturismo para el medio ambiente, como se pudo comprobar en los recorridos de campo y como también lo constatan representantes de organización ambientalistas en Los Tuxtlas es insignificante. También se puede estimar que es de menor preocupación la huella de carbono generada por los turistas que visitan los lugares ecoturísticos. De acuerdo a la información facilitada por las empresas, el porcentaje de turistas internacionales al año no sobrepasa en ningún lugar el $5 \%$, e incluso dentro de los turistas nacionales el grupo más importante en muchos lugares son personas provenientes del mismo estado de Veracruz.

Uno de los efectos positivos más importantes es la protección de áreas de selva que va desde predios muy pequeños hasta la reserva ejidal de 120 hectáreas en Adolfo López Mateos y el área protegido por La Otra Opción con una superficie de 115 hectáreas; la participación en la vigilancia ambiental de la reserva de la biosfera en general; participación en el monitoreo de las poblaciones de aves y mamíferos; reforestación en terrenos propios, igualmente desde áreas pequeñas hasta más que 20 hectáreas en los dos centros de ecoturismo privados Los Amigos y La Otra Opción, o apoyo voluntario en actividades de reforestación en otros lugares; la utilización de ecotecnologías como letrinas secas, biodigestores y paneles solares; el establecimiento de Unidades para la Conservación de la Vida Silvestre (UMA), donde se reproducen especies de animales o plantas amenazadas; y la reintroducción exitosa de la subespecie en peligro de extinción mexicana del mono aullador de manto en Nanciyaga (Lein, 2011; Neger, 2013; Vega, 2013). Recientemente se inició además un nuevo proyecto de reintroducción, de la guacamaya roja en Nanciyaga y La Otra Opción (Macías, 2015; Pérez, 2016).

Las áreas de conservación directamente relacionadas a las empresas suman 388 ha de selva y 110.5 ha de áreas de reforestación, en suma 498.5 ha. A esto se añaden áreas de selva, de reforestación o de usos agroecológicos como la siembra de café, pimienta y palmas, que pertenecen a los socios de las iniciativas comunitarias. No obstante, los representantes en las entrevistas generalmente no pudieron dar cifras exactas en cuanto a su superficie total. El ejemplo más importante en este caso es el ejido de López Mateos donde se encuentra Selva del Marinero y donde las parcelas de los ejidatarios que son sujetas a conservación y vigiladas por los integrantes del proyecto ecoturístico llegan a más que 275 ha. En los otros casos, el número es menor, aunque por ejemplo también en el caso de Los Clarines, un solo socio tiene un área de conservación de 20 ha. Por lo tanto, se puede suponer que en total la superficie protegida directamente por las empresas ecoturísticas oscila entre 750 y 800 ha.

Aunque esta superficie es relativamente pequeña si se compara con el área total de la reserva, estos terrenos forestales pueden presentar corredores importantes para la dispersión de flora y fauna (cp. Vega, 2019) y refugios donde los animales están protegidos de la cacería furtiva. En algunos casos los integrantes de las empresas ecoturísticas también realizan actividades de vigilancia y promueven la conservación en toda el área del ejido. Los resultados de estos esfuerzos se hacen notar, por ejemplo, en el regreso de especies de animales en lugares donde ya no se encontraban. Varias empresas también se empeñan directamente en la conservación de ciertas especies, mediante su reproducción en UMA.

Hasta ahora, un caso exitoso es la reintroducción de la guacamaya roja en iniciativas como la Otra Opción, Nanciyaga y recientemente también en la reserva ejidal de Benito Juárez, en cooperación con el centro ecoturístico Anolis, además que en Nanciyaga se reintrodujo al mono aullador, y de ahí se va dispersando en las áreas cercanas. De igual manera, en la iniciativa de ecoturismo Arrecifes, ubicada en una de las zonas costeras de la región, todos los socios participan en un campamento de conservación de tortugas marinas.

Por otro lado, la educación ambiental ha tenido efectos notables tanto en las familias de los integrantes de las iniciativas ecoturísticas como en la población local en general e incluso en los turistas. Varias iniciativas de ecoturismo han visto la educación ambiental muy beneficiosa para dar a conocer la realidad ambiental de la región. Un caso interesante es Nanciyaga, donde se organizan visitas escolares principalmente de primaria, para estar en contacto con el medio ambiente y aprender directamente de este (en años anteriores el número de alumnos llegó a hasta 10 mil anuales). Adicionalmente, Paré (2003) menciona que la incorporación de personas locales en el ecoturismo y los programas de capacitación relacionadas han traído consigo una mayor conciencia ambiental, nuevos conocimientos y habilidades en el uso de ecotecnologías y agroecología. 


\section{Conclusión}

El ecoturismo se ha insertado en toda la región de Los Tuxtlas, tanto dentro como fuera de los límites de la reserva de la biosfera, como parte de las estrategias de conservación ambiental, con el objetivo de involucrar a la población local. La presente investigación afirma que esta actividad efectivamente se desarrolla en este lugar de una manera que no daña el medio ambiente, así como desarrolla una conservación activa que contribuye en pequeña escala a preservar los ecosistemas de la región. Se documenta cuáles han sido los esfuerzos de conservación emprendidos en los diferentes centros ecoturísticos privados y comunitarios y se pudo evidenciar que éstos son presentes en el caso de todas las empresas.

No obstante, la magnitud de las acciones y el grado del impacto generado varía bastante, desde áreas muy pequeñas a reservas con superficies superiores a los 100 ha, que pueden representar importantes refugios y corredores para la fauna silvestre. También se han tomado acciones de reforestación, monitoreo comunitario de la biodiversidad, vigilancia ambiental y, como uno de los aspectos clave del ecoturismo, de educación ambiental y concientización de los visitantes y de los niños de las escuelas locales.

En cuanto a las grandes diferencias en los esfuerzos de conservación, estos se deben en parte a la composición de los proyectos. En los que se basan en la estructura ejidal, se disponen de grandes extensiones de terrenos forestales, mientras que la mayoría de las empresas de carácter privado tienen reservas mucho más pequeñas; sin embargo, también existen dos excepciones, con áreas de conservación y de reforestación de grandes extensiones en propiedad privada, en Los Amigos y La Otra Opción. Cabe mencionar que en la mayoría de los casos, estos espacios ya se conservaron antes del desarrollo del ecoturismo, así que la actividad ecoturística en este contexto puede ser visto más bien como de apoyo, para asegurar la persistencia a largo plazo de las áreas de conservación.

En términos generales, llama la atención de que si bien en todos los lugares analizados se realizan actividades de conservación, es escasa la vinculación entre éstas, con dos excepciones, por un lado la reintroducción de la guacamaya roja en la que participan conjuntamente Nanciyaga y Anolis, y el monitoreo de aves en el cual, bajo la organización de la dirección de la reserva de la biosfera, participan los integrantes de varios centros de ecoturismo. Es ahí donde existe un potencial importante para aumentar el impacto positivo del ecoturismo, si se lograse juntar todos los esfuerzos dispersos y de pequeña escala para integrarlos bajo una estrategia general a nivel regional.

Por otra parte, la educación ambiental realizada por las iniciativas de ecoturismo, tanto comunitarias como privadas, ha logrado reflejar un impacto en la sensibilización de la sociedad, empezando por los mismos socios que las integran. Actualmente algunas de ellas, como Manglares de Sontecomapan, Selva del Marinero, Nanciyaga, Anolis y Ecocallí, realizan actividades con grupos escolares dando talleres sobre la importancia del medio ambiente y la ecología de la región, labor que complementa a las acciones de las instituciones federales encargadas de la conservación ambiental.

De igual manera, con apoyo institucional de la Comisión Nacional para el Conocimiento y Uso de la Biodiversidad (CONABIO), La Comisión Nacional de Áreas Naturales Protegidas (CONANP) a través de La Reserva de la Biosfera Los Tuxtlas y PRONATURA a.c., se creó desde el año 2010 la "Red de Monitoreo Comunitario de Aves Huilotl Toxtlan", en la que participan 12 comunidades y entre ellas 8 centros de ecoturismo que son: Los Clarines, Selva del Marinero, El Apompal, Manglares de Sontecomapan, Ecoturismo Arqueológico Las Margaritas, Anolis, El Teterete y Nanciyaga, con el objetivo de proteger y monitorear la avifauna que existe en la región.

Por otra parte, pese a que el ecoturismo en muchas ocasiones se posiciona considerando el factor económico como un elemento fundamental debido al modelo hegemónico convencional del turismo, en la región de Los Tuxtlas y para los casos estudiados puede vislumbrarse que su esencia radica en la conservación ambiental, priorizando el manejo y cuidado de la naturaleza en un marco de sustentabilidad para la población local, desde la aparición de esta actividad hace 34 años.

En resumen, queda de aconsejar a las instituciones encargadas del cuidado del ambiente y del desarrollo de las poblaciones rurales e indígenas, y también las organizaciones no gubernamentales, de potencializar de manera estratégica el apoyo al componente del ecoturismo, dado los efectos positivos que esta actividad puede tener para la conservación, como se ha evidenciado en este trabajo en el contexto territorial de la Reserva de la Biosfera de Los Tuxtlas.

Así como, es necesario precisar que la actividad turística bajo cualquier esquema de desarrollo en la región sea ecoturismo, turismo de naturaleza, turismo rural, agroturismo, etc., nunca deberá desplazar la actividad productiva fundamental de la población local, considerándose en todo momento como una oportunidad más de contribuir a la producción, manejo y conservación del entorno natural, subsanando a nivel regional los embates ecológicos y socioeconómicos que devienen en el contexto global. 
Finalmente, a través de las experiencias que permearon nuestras investigaciones, resulta imprescindible considerar dos factores clave para los Tuxtlas: El desarrollo de proyectos integrales bajo un enfoque regional con la participación de los actores locales, a través de un acompañamiento técnico comprometido y que no encamine a los proyectos o empresas comunitarias a sobrevivir por medio de subsidios y fundamente la conservación a través del componente económico; así como, desarrollar mecanismos eficientes por las instituciones y agencias financiadoras del componente ecoturismo, para el monitoreo de los subsidios y acciones encaminadas a la conservación ambiental, pues, solo así se darán pasos correctos hacia la construcción de un verdadero ecoturismo comprometido con el medio ambiente y la sociedad.

\section{Bibliografía}

Barragán, M. 2019. 16 de abril. "Por intenso Calor 5 municipios de Veracruz, en sequía extrema: CONAGUA". Al Calor Político. Consulta: https://www.alcalorpolitico.com

Bernard, H. 2011. Research nethods in anthropology: Qualitative and quantitative approaches. Oxford. Altamira Press.

Buckley, R. 2009. "Evaluating the net effects of ecotourism on the environment: a framework, first assessment and future research". Journal of Sustainable Tourism 17(6), 643-672.

Cañada, E., Delgado, L. y Gil, H. 2006. Guía Turismo Rural Comunitario Nicaragua. 1a. ed. Managua, Nicaragua.

Ceballos-Lascuráin, H. 1998. Ecoturismo: naturaleza y desarrollo sostenible. UICN. México D.F.

CONANP. 2006. Programa de conservación y manejo de la reserva de la biosfera Los Tuxtlas. Veracruz, México.

Currie, J. J., Stack, S. H. y Kaufman, G. D. 2018. "Conservation and Education Through Ecotourism: Using Citizen Science to Monitor Cetaceans in the Four-Island Region of Maui, Hawaii”. Tourism in Marine Environments 13(2-3), 65-71.

Díaz-Carrión, I. y Neger, C. 2014. "Ecotourism in the Reserva de la Biosfera de Los Tuxtlas (Veracruz, Mexico)". Athens Journal of Tourism, 1(3), p. 191-202.

da Silva, M., Regina, M., Correira, C., Guedes, R. y Neiva, M. 2018. "Contribución del ecoturismo a la conservación del guacamayo rojo (arara vermelha) en una reserva de Brasil". Estudios y Perspectivas en Turismo 27, 158-177.

de la Maza, J., Carabias, J., Ruiz, L., Mastretta, A. y Valadez, V. 2015. Ecoturismo para la conservación: Bases para el desarrollo ecoturístico en el municipio Marqués de Comillas, Selva Lacandona, Chiapas. Natura y Ecosistemas Mexicanos, A.C., México.

Fangfang, S., Weaver, D., Zhao, Y., Huang, M.-F., Tang, C. y Liu, Y. 2019. “Toward an ecological civilization: Mass comprehensive ecotourism indications among domestic visitors to a Chinese wetland protected area". Tourism Management 70, 59-68.

Fennell, D. A. 2008. Ecotourism. Tercera edición. Abingdon (Reino Unido): Routledge.

Fletcher, R. 2018. "Ecotourism". En: Castree, N., Hulme, M. y Proctor, J. D. (eds): Companion to Environmental Studies (pp. 491-494). Abingdon y Nueva York: Routledge.

Fletcher, R. y Neves, K. 2012. "Contradictions in Tourism: The Promise and Pitfalls of Ecotourism as a Manifold Capitalist Fix". Environment and Society 3(1), 60-77.

García, María D. 2010. The Potential of Community-based Ecotourism Projects as Environmental-Education-for-Sustainability Centres. A case study of Los Tuxtlas Community-based Ecotourism Network (Mexico). Tesis de maestría, Londres: King's College.

García de Fuentes, A., Jouault, S. y Romero, D. 2015. Atlas de Turismo Alternativo en la Península de Yucatán. CINVESTAV-UADY. Mérida, Yucatán.

Geffroy, B., Sadoul, B. y Ellenberg, U. 2017. "Physiological and Behavioral Consequences of Human Visitation”. En Blumstein, D. T., Geffroy, B., Samia, D. S. M. y Bessa, E. (eds.), Ecotourism's Promise and Peril: A Biological Evaluation (p. 9-27). Cham (Suiza): Springer.

Gómez, F. J. 2017, "15 de abril. Cambio climático y degradación ambiental, combinación peligrosa para el campo". La Jornada del Campo 115. Consulta en: https://www.jornada.com.mx/2017/04/15/ cam-climatico.html

González Kuk G. 2018. Perspectivas y contribuciones del ecoturismo a la conservación ambiental en la región de los Tuxtlas Veracruz, México. Tesis de maestría. Colegio de Postgraduados, Córdoba, Veracruz, México. 
González Kuk G. 2018. "Historia socioambiental, movimientos étnicos y conservación en la región de los Tuxtlas, Veracruz, México". Devenir revista de estudios culturales y regionales 34. P. 199-217.

Guzmán, M., Figueroa, F. y L., Durand 2013. "Ecología política y ecoturismo en México: reflexiones desde la Huasteca Potosina y la Selva Lacandona” (pp. 29-579). En: Guzmán, M. y D. Juárez (eds.), En busca del ecoturismo. Casos y experiencias del turismo sustentable en México, Costa Rica, Brasil y Australia. EÓN-El Colegio de San Luis A.C. México.

Higham, J. 2007. "Ecotourism: competing and conflicting schools of thought". En Higham, J. (ed.), Critical issues in ecotourism: understanding a complex tourism phenomenon (p. 1-19), Elsevier Butterworth-Heinemann, Oxford. Burlington.

Honey, M. 2008. Ecotourism and sustainable development: Who owns paradise? Segunda Edición. Washington (EEUU): Island Press.

Huaraca, L. E., Paladines, G. V. y Suárez, J. E. 2017. "Gestión del turismo comunitario como alternativa de desarrollo local. Comunidad Yunguilla. Quito”. Revista Publicando 4(11/2). 427-441.

Juárez, L. E. 2016. Proceso de adopción, adaptación y apropiación de una propuesta de ecoturismo en cuatro comunidades en la Reserva de la Biosfera Los Tuxtlas. Tesis de maestría. Universidad Nacional Autónoma de México.

IUCN, International Union for Conservation and Nature 1996. Resoluciones y Recomendaciones Congreso Mundial de la Naturaleza Montreal, Canadá. Congreso Mundial de la Naturaleza. Reino Unido.

K.C., A. 2016. "Ecotourism and its Role in Sustainable Development of Nepal". En Butowski, L. (ed.). Tourism: From Empirical Research Towards Practical Application (p. 31-60). Rijeka (Croacia): InTech.

Kiss, A. 2004. "Is community-based ecotourism a good use of biodiversity conservation funds?" Trends in Ecology \& Evolution 19(5), p. 232-237.

Lara, D., Rojas, C. Y Velásquez-Tibatá, J. 2016. “Turismo de Naturaleza: Oportunidad de desarrollo de las comunidades locales". En Biodiversidad. Reporte Humbolt ORG. Bogotá, Colombia.

Lein, Johanna T. W. 2011. Akzeptanzschaffung für Biosphärenreservate durch Ökotourismus - eine Analyse der Wahrnehmung der Biosphärenreservates Los Tuxtlas, Mexiko, durch lokale Ökotourismusbetriebe. Tesis de Maestría. Greifswald: Universidad de Greifswald.

Liu, Z. H. 2003. "Sustainable tourism development: A critique". Journal of Sustainable Tourism 11(6). 459-475.

Macías, Carlos M. 2015. Valores biológicos y culturales para el desarrollo del ecoturismo ornitológico de Los Tuxtlas. Tesis de licenciatura. México: Universidad Nacional Autónoma de México.

Magio, K. O., Guillén, E. \& E. M. Carballo. 2017. "Ecoturismo y conservación en el ejido Ruiz Cortines, Los Tuxtlas". Teoría y Praxis 22. 159-195.

Marchena, M.; Rosabal, P.; Salinas, E.; Fernández, B.; Dorado, Y. 1993. "Planificación y Desarrollo Del Ecoturismo". Estudios Turísticos. 119120. 39-58.

Marzouki, M., Froger, G. y J. Ballet 2012. "Ecotourism versus Mass Tourism. A Comparison of Environmental Impacts Based on Ecological Footprint Analysis". Sustainability 4(1). 123-140.

Mbaiwa, J. E. 2015. "Ecotourism in Botswana: 30 years later". Journal of Ecotourism 14 (2-3). 204-222.

Melgarejo, J. 1949. Historia de Veracruz. Gobierno de Xalapa, Veracruz. 528 p.

Mossaz, A., Buckley, R. C. y Castley, J. G. 2015. "Ecotourism contributions to conservation of African big cats". Journal for Nature Conservation 28. 112-118.

Neger, Christoph 2013. Die Rolle des Ökotourismus für die Nachhaltige Entwicklung einer Region: Fallstudien aus Los Tuxtlas/Mexiko. Saarbrücken: Akademikerverlag.

Neger, Christoph 2019. Actores y redes sociales involucrados en el desarrollo del ecoturismo en la Reserva de la Biosfera de Los Tuxtlas, Veracruz. Tesis de doctorado. México: Universidad Nacional Autónoma de México.

Olaya, V. 2014. Sistemas de Información Geográfica. Creative Common Atribución. Ed. Primera. 854 p.

Orgaz, F. 2014. "El Ecoturismo en los Humedales: Análisis de las Potencialidades de República Dominicana”. rosa dos ventos-Turismo e Hospitalidade, 61. 4-18.

Orgaz, F.; Castellanos, M. 2013. "Conceptualización y consideraciones en torno al ecoturismo". Revista de Investigación En Turismo y Desarrollo Local. 615, 1-12.

Paré, Luisa 2003 "El ecoturismo comunitario y la gestión ambiental”. En Paré, Luisa y Lazos, Elena (Eds.), Escuela rural y organización comunitaria: instituciones locales y manejo ambiental (pp. 261-311). México: Plaza y Valdés.

Paré, L.; Fuentes, T. 2007. Gobernanza Ambiental Y Políticas Públicas En Áreas Naturales Protegidas: Lecciones Desde Los Tuxtlas. UNAM. Ed. Primera ed. México D.F. 265 p. 
Pérez, Juan L. F. 2016 Evaluación del potencial de ecoturismo en la Reserva de la Biosfera de Los Tuxtlas, Veracruz como alternativa de sustentabilidad al uso actual del suelo. Tesis de licenciatura, México: Universidad Nacional Autónoma de México.

Piñar-Álvarez, A. 2011. Gobernanza ambiental en destinos turísticos de áreas naturales protegidas: Reservas de la Biosfera Los Tuxtlas (Veracruz, México) y Sierra Nevada-La Alpujarra (Andalucía, España). Xalapa: El Colegio de Veracruz.

Puertas, I. 2006. "Ecoturismo en las reservas de biosfera: análisis del ecoturista en Bañados del Este Uruguay y Cabo de Gata-Níjar España”. Estudios Turísticos.170169, 183-200.

Ramírez, F. 1999. Flora y vegetación de la Sierra de Santa Marta, Veracruz. Universidad Autonóma De México.

Rivera, M. 2010. Turismo activo en la naturaleza y espacio de ocio en Andalucia: Aspectos territoriales, políticas públicas y estrategias de planificación. Universidad de Córdoba.

Rosas-Baños, M. y Correa-Holguín, D. A. 2016. "El ecoturismo de Sierra Norte, Oaxaca desde la comunalidad y la economía solidaria”. Agricultura, sociedad y desarrollo 13(4). 565-584.

Salazar, M. 2020. "Crisis en campo hace migrar a agrónomos y veterinarios". Diario de Xalapa. Consulta en: https://www.diariodexalapa.com.mx/local/crisis-en-campo-hace-migrar-a-agronomos-y-veterinarios-panuco-cosecha-ganaderos-maiz-frijol-4845528.html

Secretaría de Turismo 2016 Diagnóstico Grupo Intersectorial de Turismo de Naturaleza [SECTUR, SEMARNAT, FONATUR, UNAM, ICTUR, SUSTENTUR]. México.

Secretaría de Gobernación. 2019. 19 de diciembre. DECLARATORIA de Desastre Natural por la presencia de sequía severa ocurrida del 1 de mayo al 30 de noviembre de 2019 en 68 municipios del Estado de Veracruz de Ignacio de la Llave. Diario Oficial de la Federación 19/12/2019. Secretaría de Gobernación, México.

Serulle, H.1999. “El Bosco Y Darwin: Pioneros Del Ecoturismo”. En F. C. Y Arte Ed., Ecoturismo Y Desarrollo Sostenible En República Dominicana El Caribe Y El Mundo. 350 p.

Simmons, D. 1999. "Eco-tourism: product or process". Sciences-New York, 1989, 1-9.

Shannon, G., Larson, C. L., Reed, S. E., Crooks, K. R. y Angeloni, L. M. 2017. "Ecological Consequences of Ecotourism for Wildlife Populations and Communities". En Blumstein, D. T., Geffroy, B., Samia, D. S. M. y Bessa, E. (eds.), Ecotourism's Promise and Peril: A Biological Evaluation (p. 29-46). Cham (Suiza): Springer.

Vargas, G. 2004. "De brujos y curanderos". Ulúa Revista de sociedad y cultura. v. 2. no. 4.127-147

Vanelli, K., Hampton, M. P., Namgail, T. y Black, S. A. 2019. "Community participation in ecotourism and its effect on local perceptions of snow leopard (Panthera uncia) conservation". Human Dimensions of Wildlife 24 (2). 180-193.

Vega, V. 2013 Percepción de la sustentabilidad del ecoturismo bajo el enfoque de agroecosistemas: El caso de la reserva de la biosfera Los Tuxtlas, Veracruz. Tesis de maestría. Xalapa: Colegio de Posgraduados.

Vega, V. 2019 Contribución de las UMA extensivas a la Conservación y Conectividad del Paisaje de Los Tuxtlas, Veracruz. Tesis de doctorado. Xalapa: Centro de Investigaciones Tropicales de la Universidad Veracruzana.

Voumard, M. 2019. "Promises and pitfalls of ecotourism: patterns from a literature review". Investigaciones Turísticas 17. 1-23.

Wearing, S. y J. Neil 1999. Ecotourism: Impacts, Potentials and Possibilities. Oxford (Reino Unido): Elsevier Butterworth-Heinemann, Oxford.

\section{Notas}

1 En el contexto nacional, el turismo de naturaleza o en las modalidades que administrativamente se han integrado como Turismo de Aventura, Turismo Rural y Ecoturismo (SECTUR, 2016) y se ha buscado desarrollar la actividad turística en diferentes contextos geográficos siguiendo los componentes recreativos y económicos.

2 Cabe mencionar que existen nociones de mayor complejidad acerca del ecoturismo, que argumentan que este concepto establece una propia filosofía y esboza un modelo de desarrollo particular (Simmons, 1999).

$3 \quad$ Esto después de que en 1937 se haya determinado por Decreto Federal la Zona Protectora Forestal Vedada de la Cuenca Hidrográfica del Lago de Catemaco, con una protección de 28,500 ha, principalmente para el resguardo del cuerpo hidrológico.

4 Pese a que las UMA permiten la realización de las actividades ecoturísticas, no es su finalidad u objetivo perseguir actividades recreativas o de ocio, sino su prioridad es la conservación ambiental, resguardo de especies de flora y/o fauna, así como la educación ambiental. 
5 La educación ambiental, un aspecto clave del ecoturismo genuino, es presente en muchos de los centros turísticos mencionados en los estudios. se practica, de acuerdo con un estudio de García (2010), especialmente dirigida hacia los visitantes y, en menor medida, los niños de escuelas locales. Al contrario, es escasa la educación ambiental hacia personas adultas locales que no están involucradas directamente en los centros ecoturísticos.

$6 \quad$ A nivel nacional el abandono de iniciativas de ecoturismo se ha reportado, por ejemplo, de la Huasteca Potosina y la Selva Lacandona (Guzmán et al., 2013) y de la península de Yucatán, donde García de Fuentes et al. (2015) reportan 48 casos de empresas comunitarias de turismo alternativo abandonados, aparte de otras 63 empresas (el $41.2 \%$ de las empresas de turismo alternativo activas en este momento en las comunidades indígenas de la península) que se mantenían solamente debido a los subsidios otorgados por las instituciones gubernamentales.

7 Véase apartado Ecoturismo: conceptualización y praxis 